2016-08

\title{
A comparison of energy consumption between the use of a walking frame, crutches and a Stride-on rehabilitation scooter
}

\section{Patel, N}

http://hdl.handle.net/10026.1/5364

10.1016/j.foot.2016.04.002

The Foot

Elsevier

All content in PEARL is protected by copyright law. Author manuscripts are made available in accordance with publisher policies. Please cite only the published version using the details provided on the item record or document. In the absence of an open licence (e.g. Creative Commons), permissions for further reuse of content should be sought from the publisher or author. 
A Comparison of energy consumption between the use of a walking frame, crutches and a Stride-on rehabilitation scooter

Authors:

${ }^{1}$ Mr Nimesh Patel, MRCS

${ }^{1}$ Mr Timothy Batten, MRCS

${ }^{1} \mathrm{Mr}$ Andrew Roberton, MRCS

${ }^{3} \mathrm{Mr}$ Doyo Enki

Mr Guy Wansbrough FRCS (Trauma and Orthopaedics)

${ }^{1}$ Mr James Davis, FRCS (Trauma and Orthopaedics)

Institution:

${ }^{1}$ Department of Trauma and Orthopaedics, Torbay Hospital, Torquay, TQ2 7AA, UK

Corresponding author:

Mr Nimesh Patel

Dr.nimeshpatel1@gmail.com

$+447939941883$

Declarations:

No financial disclosures

No conflicts of interest

All authors were fully involved in the study and preparation of the manuscript. The material has not been and will not be submitted for publication elsewhere. 


\section{Brief Summary}

- The energy consumption ambulating with a stride-on knee walker has not been investigated previously, and no energy efficiency studies have been carried out using Cardio pulmonary exercise testing

- The use of a stride-on knee walker requires less energy compared to ambulation with crutches or a frame

- This can be used in post-operative patients to aid rehabilitation in patients with reduced upper body strength or poor cardiovascular reserve 


\title{
A Comparison of energy consumption between the use of a walking frame,
}

\section{crutches and a Stride-on rehabilitation scooter}

\author{
ABSTRACT \\ Background \\ Following foot and ankle surgery, patients may be required to mobilise non-weight \\ bearing, requiring a walking aid such as crutches, walking frame or a Stride-on \\ rehabilitation scooter, which aims to reduce the amount of work required. The energy \\ consumption of mobilising using a Stride-on scooter has not previously been \\ investigated, and we aim to establish this. \\ Methods \\ Ten healthy volunteers ( 5 male: 5 female) aged $20-40$ years mobilised independently, \\ then with each mobility device for 3 minutes at $1 \mathrm{~km} / \mathrm{h}$ on a treadmill, with rest periods, \\ whilst undergoing Cardio-Pulmonary Exercise Testing (CPET). \\ Oxygen consumption ( $\left.\mathrm{VO}_{2}\right)$, carbon dioxide excretion $\left(\mathrm{VCO}_{2}\right)$, minute ventilation (MV), \\ respiratory rate (RR) and pulse (HR) were measured at baseline, and after 3 minutes of \\ walking, without and with all 3 devices. \\ Wilcoxon signed rank test was carried out to calculate significance with non-parametric \\ values with Bonferroni correction. \\ Results \\ Three-point crutch mobilisation demonstrated significant increases in $\mathrm{VO}_{2}(0.7 \mathrm{~L}), \mathrm{VCO}_{2}$ \\ (0.7L), MV (16.7L/min), pulse (24.8bpm) and RR (11.4 breaths $/ \mathrm{min})$ compared to \\ walking ( $p<0.05)$. Mobilisation with a frame produced significant $(p<0.05)$ increases \\ compared to walking; $\mathrm{V0}_{2}(0.7 \mathrm{~L}), \mathrm{VCO} 2(0.7 \mathrm{~L}), \mathrm{MV}(18.3 \mathrm{~L} / \mathrm{min})$, pulse $(35.9 \mathrm{bpm})$, and \\ RR (11.7 breaths/min). Tests using the Stride-on demonstrated no significant increase
}


compared to walking with regards to $\mathrm{VO}_{2}(0.1 \mathrm{~L} ; p=0.959), \mathrm{VCO}_{2}(0.2 \mathrm{~L} ; p=0.332)$, Pulse (10.1bpm; $p=0.575$ ), and RR ( $4.7 \mathrm{breaths} / \mathrm{min} ; p=0.633$ ). The MV was significantly higher compared to walking $(4.3 \mathrm{~L} / \mathrm{min} ; p<0.05)$.

Discussion

Energy required for unit distance ambulation with a Stride-on device is similar to walking, and significantly lower than with a walking frame in single legged stance and three-point crutch mobilisation. This justifies its use as part of routine practice aiding early mobilisation of patients requiring restricted weight bearing or single legged weight bearing, especially in those with reduced cardio-pulmonary reserve as it is less physiologically demanding and does not rely on upper body strength.

Key words: Energy efficiency, energy consumption, walking aids, lower limb injuries, stride-on, frame, crutches 


\section{INTRODUCTION}

Medical rehabilitation requires active participation and work on the part of the patient seeking to regain the capability of ambulating. Effective rehabilitation programmes must attempt to increase the patient's ability to perform work and concurrently decrease the amount of work required. The former goal is achieved through exercise and rehabilitation training, and the latter by use of adaptive devices or environmental manipulation(1).

Following foot-and-ankle surgery, many patients will need to mobilise without bearing weight through their operated limb. This requires the use of a mobility aid. There are a number of devices available to patients to aid rehabilitation following foot-and-ankle orthopaedic surgery or lower limb surgery. These include crutches, walking frames and more recently innovative devices such as the 'Stride-On' knee walker. The 'Stride-on' knee walker is a five wheeled, patient-propelled, mobility scooter designed specifically for patients who have undergone foot-and-ankle surgery. Its key design features include four stabilising wheels, a fifth wheel for steering, which is attached to a steering column, a knee support for the patient to rest the affected knee and shin on, and a shopping basket(2). Three-point ambulation with crutches has previously been shown to double the energy expended by patients compared with normal walking $(3,4)$. It has also been demonstrated that patients with spinal cord injuries have a higher energy consumption and oxygen cost, with a lower walking velocity compared with able-bodied control subjects, and within this group, mobilising with three point crutch ambulation is more energy efficient compared to a walker(5).

To date, there is no record in the literature as to the energy consumption of a patient using a 'Stride-on' device. It has been shown however that a two-wheeled mobility 
scooter, designed for African amputees, which is significantly different in design to the 'Stride-on', reduced energy consumption by $60 \%$ compared with three-point crutch ambulation(6).

We therefore seek to investigate the difference in energy consumption between a walking frame and three-point ambulation with crutches compared to the Stride-on rehabilitation scooter. 
AIM

To investigate the difference in energy consumption with the use of a Stride-on

rehabilitation scooter compared with the use of crutches (three point ambulation) or a

walking frame in physically healthy individuals to identify the suitability of its use.

\section{NULL HYOPTHESIS}

There is no difference in the amount of energy consumed when mobilising with a Stride-on scooter compared to a walking frame or crutches. 


\section{MATERIALS AND METHODS}

We recruited ten healthy and able-bodied volunteers aged between $20-40$ years. They were all able to give valid consent, and none had any history of lower limb injury in the past or any difficulty mobilising. They took no long-term medication and were all nonsmokers.

All patients underwent baseline measurements of height, weight, heart rate (HR), resting oxygen saturations (SATS) and resting blood pressure (BP).

We measured energy consumption using Cardio-Pulmonary Exercise Testing (CPET) whilst the subjects were asked to mobilise on a standard treadmill (Figure 1).

A pilot trail was carried out to calculate ideal walking speed and time that could be transferred to all 3 mobility aids safely, without tiring significantly and all subjects were required to mobilise at a speed of $1 \mathrm{~km} / \mathrm{h}$ for a time of 3 minutes a zero inclination, whilst being on the CPET monitor.

CPET is a functional assessment of cardiopulmonary reserve, and as been used to assess elite athletes as well as assessing fitness for surgery. The ability to perform exercise is related to the cardiopulmonary systems ability to supply oxygen and remove carbon dioxide. This requires adequate pulmonary ventilation, gas exchange, gas and substrate transport in the blood and uptake and utilisation at a cellular level. CPET is a measure to reflect this whole process(7).

The variables measured include oxygen consumption $\left(\mathrm{VO}_{2}\right)$, carbon dioxide excretion $\left(\mathrm{VCO}_{2}\right)$, minute ventilation (MV) and respiratory rate (RR). The $\mathrm{VO}_{2}$ is the product of the cardiac output and arterio-venous oxygen difference and therefore also reflects cellular aerobic oxidation of glycogen and fatty acids in the mitochondria as adenosine triphosphate is sourced. The $\mathrm{VCO}_{2}$ should then reflect the $\mathrm{VO}_{2}$ changes as long as the exercise is within the anaerobic threshold, and there is no cardiopulmonary disease. 
This is therefore a good and sensitive marker of energy consumption and reflects all aspects of cardiopulmonary function( 8,9$)$.

The setup involves walking on the treadmill whilst being connected to a pulse-oximeter at the set speed. Inspired and expired gases are sampled by a metabolic cart via a mouthpiece whilst a nose clip is worn, allowing oxygen consumption and carbon dioxide excretion to be measured by the calibrated machine. Subjects all initially performed the walk test and then sequentially perform tests with a narrow walking frame, which was adjusted to fit the treadmill belt, followed by three point mobilisation using crutches, and finally on the Stride-on mobility scooter (Model E3SL, StrideOn UK Ltd, Somerset, UK), which was locked in straight steer for safety (Figure 2). They mobilised on their dominant leg and all had a 30-minute rest in between tests to allow for a full recovery, preventing any influence on subsequent runs.

All data was collected on a spreadsheet using Microsoft Excel (Microsoft, Redmond, WA, USA), and statistical analysis was carried out using SPSS software (Version 20.0, IBM Corporation, Chicago Illinois). Wilcoxon signed rank test was carried out to compare non-parametric values and test significance, along with the median, using a 95\% confidence interval. A Bonferroni correction was also carried out to counteract the effect of multiple comparisons.

The study had been reviewed and approved by the South Devon Healthcare NHS Foundation Trust (SDHNFT) Research and Development Department. The trial was conducted in compliance with the principles of the Declaration of Helsinki (1996), the principles of good clinical practice and in accordance with all applicable regulatory requirements including but not limited to the Research Governance Framework for Health \& Social Care. 


\section{RESULTS}

Ten subjects underwent testing, including 5 males and 5 females. The age range was 24

- 38 years. None of the subjects had any history of cardiovascular pathology, took no regular medication, did not smoke, and had no mobility issues or foot and ankle pathology. All 10 completed the full test cycle. The Body Mass Index (BMI) ranged from $17.1-33.5 \mathrm{~kg} / \mathrm{m}^{2}$.

Table 1 shows all the baseline characteristics and measurements at rest for each individual. Table 2 summarises the results for the tests utilising each walking aid and these results have been compared to walking for the individual subject independently. Both median and mean values have been included to demonstrate the homogeneity of the results.

Due to the small numbers, the median was used to calculate significance using a nonparametric method. We set expected changes of $\mathrm{VO}_{2}$ and $\mathrm{VCO}_{2}$ at $0.1 \mathrm{~L}$, minute ventilation at $0 \mathrm{~L} / \mathrm{min}$, pulse at 10 beats $/$ minute, and respiratory rate at 5 breaths/minute.

These results demonstrate that the use of three-point crutch mobilisation demonstrated significantly higher median changes in $\mathrm{VO}_{2}(0.7 \mathrm{~L} ; 140 \%), \mathrm{VCO}_{2}(0.6 \mathrm{~L} ; 150 \%), \mathrm{MV}$ (17.3L/min; $142 \%)$, pulse (19bm; $23 \%$ ) and RR ( 7.5 breaths $/ \mathrm{min} ; 54 \%$ ) compared to 3 minutes of walking independently $(p<0.05)$. Mobilisation with a frame also produced similar increases compared to walking; $\mathrm{VO}_{2}$ (0.7L; $\left.140 \%\right), \mathrm{VCO}_{2}$ (0.8L; $\left.200 \%\right), \mathrm{MV}$ (19.9L/min; $163 \%)$, pulse (29.5bpm; $36 \%)$, and RR (10.0 breaths $/ \mathrm{min} ; 71 \%)$. Even when applying the post-hoc Bonferroni correction for multiple comparisons, the p-value becomes set at 0.017 . This means the parameters of $\mathrm{VO}_{2}, \mathrm{VCO}_{2}, \mathrm{MV}$, and $\mathrm{RR}$ remain significantly higher using crutches and a frame compared to walking. 
Tests using the Stride-on device demonstrated no significant increase compared to walking over the test period with regards to $\mathrm{VO}_{2}(0.1 \mathrm{~L}), \mathrm{VCO}_{2}(0.1 \mathrm{~L})$, Pulse $(7 \mathrm{bpm})$, and RR (4.0 breaths/min). The Minute Ventilation was significantly higher with a Stride-on compared to walking $(4.9 \mathrm{~L} / \mathrm{min})$. 


\section{DISCUSSION}

Our results demonstrate that the energy expenditure whilst mobilising with a Stride-on device is similar to walking, and significantly lower than mobilising with a walking frame in single legged stance and three-point crutch mobilisation. This justifies the use of a Stride-on device as a part of routine practice aiding early mobilisation of patients requiring restricted weight bearing or single legged weight bearing. Restricted weight bearing is used frequently as part of orthopaedic rehabilitation. It is not only indicated in lower extremity fractures, but also so rheumatoid conditions, vascular or diabetic conditions, as well as being part of a post operative regime. Balance problems, decreased cardiovascular endurance and inadequate upper extremity strength can all hinder patients required to perform non-weight bearing as part of rehabilitation(10). This can lead to adverse physiological as well as psychological effects as it may result in the patient remaining bed bound or confined to bed to chair activity only. This also has a wider impact on social care when considering safe discharge of patients from hospital, which can mean a longer hospital stay or requirement of interim placements, with its own added economic impact. There may also be a reduced compliance with instructions if patients feel they are unable to achieve non-weight bearing, which affects their clinical outcome.

The Stride-on device does not rely on maintaining stability using upper body strength and we have demonstrated less physiological demand on the cardiopulmonary system. Therefore, not only can patients mobilise using this device more easily, but also they can potentially travel further and regain more independence.

There is clearly a physiological benefit with the Stride-on device over crutches or a walking frame; there was no significant increase in pulse rate or respiratory rate compared to walking with no aids. This makes it more appropriate in those older 
patients who have reduced cardiopulmonary reserve and quite possibly will have multiple co-morbidities. There is also a metabolic advantage, since the $\mathrm{VO}_{2}$ and $\mathrm{VCO}_{2}$ are lower; it represents less energy consumption and ATP liberation at a cellular level, which again aids recovery and mobility, and therefore makes it an attractive option for aiding rehabilitation.

A previous study in healthy volunteers also reported higher energy consumption when mobilising non-weight bearing with a walking frame, however they demonstrated no physiological difference between non-weight bearing and touch-weight bearing. Therefore we do not feel allowing to touch-weight bear would be an adequate solution(11).

Minute ventilation was significantly higher with the Stride-on compared to walking. An explanation for this would be that a difference of $0 \mathrm{~L} / \mathrm{min}$ from the median compared to walking was utilised in the calculation. There is obviously going to be an increase here compared to walking, however the numerical values are far less compared to a walking frame and crutches.

Elderly patients with balance issues may still have difficulty co-ordinating with the device, and it may take time for these patients to mobilise safely with this device. There is therefore a learning effect with the device, and with time would become easier to use and co-ordinate.

Although not justifiable to provide for all patients, a small fleet can be kept for appropriate patients from the hospital (provided they are returned), or the patients can themselves purchase or rent from the company directly. The cost of a new device is $£ 260$, and to rent for a week costs $£ 15.50$ (prices correct as of May 2015)(2). Although a significantly higher cost compared to crutches or a frame, there are potential clinical 
benefits to the patient of early mobilisation, and economical benefits to the hospital if it facilitates early discharge.

This study represents a unique design to study the effects of different mobility aids on a healthy population. Compared to other studies, we have also shown higher energy expenditure with 3-point mobilisation. However, with our study, we have utilised the full array of mobility devices for comparison, and with the use of CPET, a very accurate and informative measure of physiological parameters to study energy expenditure and physiological response to exercise, which is used routinely in clinical practice.

\section{Study limitations}

The small number of subjects included in the study is a clear limitation, though adjustments were made with the analysis of the data to adjust for this. We also only tested on fit and healthy young volunteers, and it can be argued that maybe these findings cannot be extrapolated to more elderly patients. However, our study population allows correcting for as few confounding factors as possible.

It could be hypothesised that if we replicated non-weight bearing on a single limb within a fabricated lower leg cast, the results may be more transferrable to clinical practice. However previous gait studies using unilateral non-weight bearing swing through gait with and without a cast showed no change it oxygen consumption(12).

\section{Brief Summary}

- The energy consumption ambulating with a stride-on knee walker has not been investigated previously, and no energy efficiency studies have been carried out using Cardio pulmonary exercise testing

- The use of a stride-on knee walker requires less energy compared to ambulation with crutches or a frame

- This can be used in post-operative patients to aid rehabilitation in patients with reduced upper body strength or poor cardiovascular reserve 


\section{REFERENCES}

1. The Physiological Basis of Rehabilitation Medicine [Internet]. Elsevier Science; 2013 [cited 2015 Apr 20]. 782 p.

2. StrideOn Knee Walker [Internet]. [cited 2015 May 8]. Available from: https://www.strideon.co.uk/

3. Waters RL, Campbell J, Perry J. Energy cost of three-point crutch ambulation in fracture patients. J Orthop Trauma [Internet]. 1987 Jan [cited 2015 May 8];1(2):170-3.

4. Fisher S V, Patterson RP. Energy cost of ambulation with crutches. Arch Phys Med Rehabil [Internet]. 1981 Jun [cited 2015 May 8];62(6):250-6.

5. Ulkar B, Yavuzer G, Guner R, Ergin S. Energy expenditure of the paraplegic gait: comparison between different walking aids and normal subjects. Int J Rehabil Res [Internet]. 2003 Sep [cited 2015 Apr 22];26(3):213-7.

6. McCahill J, Stebbins J, Bates J, Batchelor A, Church J, Lavy C. The African Disability Scooter: preliminary analysis of a new mobility aid. Disabil Rehabil Assist Technol [Internet]. 2009 Sep [cited 2015 May 8];4(5):353-6.

7. Albouaini K, Egred M, Alahmar A, Wright DJ. Cardiopulmonary exercise testing and its application. Postgrad Med J [Internet]. 2007 Nov [cited $2015 \mathrm{Mar}$ $10] ; 83(985): 675-82$.

8. Balady GJ, Arena R, Sietsema K, Myers J, Coke L, Fletcher GF, et al. Clinician's Guide to cardiopulmonary exercise testing in adults: a scientific statement from 
the American Heart Association. Circulation [Internet]. 2010 Jul 13 [cited 2015 May 8];122(2):191-225.

9. CPET | Cardiopulmonary Exercise Testing | Cardiopulmonary Exercise Test | Essex Cpex [Internet]. [cited 2015 May 8].

10. Kathrins BP, O'Sullivan SD. Cardiovascular responses during nonweight-bearing and touchdown ambulation. Phys Ther [Internet]. 1984 Jan [cited 2015 May 8];64(1):14-8.

11. Westerman RW, Hull P, Hendry RG, Cooper J. The physiological cost of restricted weight bearing. Injury [Internet]. Elsevier; 2008 Jul 7 [cited 2015 May 8];39(7):725-7.

12. Waters RL, Campbell J, Thomas L, Hugos L, Davis P. Energy costs of walking in lower-extremity plaster casts. J Bone Joint Surg Am [Internet]. 1982 Jul [cited 2015 May 8];64(6):896-9. 
Appendix

Figure 1: Cardio Pulmonary Exercise Testing Machine

Figure 2: StrideOn Scooter on Treadmill

\begin{tabular}{|c|c|c|c|c|c|c|c|c|c|c|}
\hline Age & Gender & $\begin{array}{l}\text { Height } \\
\text { (m) }\end{array}$ & $\begin{array}{l}\text { Weight } \\
\text { (kg) }\end{array}$ & $\begin{array}{l}\text { BMI } \\
\left(\mathrm{kg} / \mathrm{m}^{2}\right)\end{array}$ & $\begin{array}{l}\text { BP } \\
(\mathrm{mm} / \mathrm{Hg})\end{array}$ & Pulse & $\begin{array}{l}\text { V02 } \\
\text { (L) }\end{array}$ & $\begin{array}{l}\text { VCO2 } \\
\text { (L) }\end{array}$ & $\begin{array}{l}\text { MV } \\
\left(\mathbf{L}_{\text {min }}\right)\end{array}$ & RR \\
\hline 33 & $\mathrm{~F}$ & 1.620 & 87.8 & 33.5 & $135 / 90$ & 55 & 0.31 & 0.25 & 12.0 & 14 \\
\hline 38 & $\mathrm{~F}$ & 1.810 & 56.0 & 17.1 & $108 / 73$ & 83 & 0.22 & 0.19 & 7.0 & 13 \\
\hline 26 & $\mathrm{~F}$ & 1.690 & 59.5 & 20.8 & $132 / 93$ & 60 & 0.24 & 0.27 & 8.2 & 9 \\
\hline 25 & $\mathrm{~F}$ & 1.670 & 55.8 & 20.0 & $113 / 73$ & 70 & 0.18 & 0.20 & 7.0 & 7 \\
\hline 24 & $M$ & 1.810 & 92.0 & 28.1 & $155 / 88$ & 96 & 0.24 & 0.20 & 6.0 & 4 \\
\hline 28 & $M$ & 1.870 & 87.0 & 24.9 & $137 / 85$ & 72 & 0.30 & 0.40 & 15.4 & 9 \\
\hline 27 & $M$ & 1.760 & 83.0 & 26.8 & $134 / 87$ & 51 & 0.29 & 0.23 & 7.2 & 12 \\
\hline 29 & M & 1.870 & 79.0 & 22.6 & $157 / 79$ & 68 & 0.36 & 0.40 & 13.7 & 10 \\
\hline 28 & $M$ & 1.610 & 67.0 & 25.8 & $115 / 80$ & 72 & 0.21 & 0.16 & 8.0 & 12 \\
\hline 27 & $\mathrm{~F}$ & 1.610 & 56.0 & 21.6 & $115 / 80$ & 76 & 0.21 & 0.16 & 6.6 & 12 \\
\hline
\end{tabular}

Table 1: Baseline Characteristics

\begin{tabular}{|c|c|c|c|c|c|c|}
\hline & & Pulse & V02 (L) & VCO2 (L) & MV (L/min) & $\mathbf{R R}$ \\
\hline \multirow[t]{2}{*}{ Walking } & Mean (SD) & $83.7(12.9)$ & $0.5(0.1)$ & $0.4(0.2)$ & $14.5(6.3)$ & $12.1(4.9)$ \\
\hline & Median (IQR) & $82.0(14.8)$ & $0.5(0.2)$ & $0.4(0.1)$ & $12.2(2.9)$ & $14.0(8.5)$ \\
\hline \multirow[t]{3}{*}{ Crutches } & Mean (SD) & $108.5(23.1)$ & $1.2(0.3)$ & $1.1(0.4)$ & $31.2(8.8)$ & $23.5(6.6)$ \\
\hline & Median (IQR) & $101.0(29)$ & $1.2(0.1)$ & $1.0(0.1)$ & $29.5(3.9)$ & $21.5(7.8)$ \\
\hline & p-value (VS walking) & $0.037^{\star}$ & $0.005^{\star}$ & $0.007^{\star}$ & $0.007^{\star}$ & $0.011^{\star}$ \\
\hline \multirow[t]{3}{*}{ Frame } & Mean (SD) & $119.6(25.0)$ & $1.2(0.3)$ & $1.1(0.3)$ & $32.8(7.1)$ & $23.8(4.3)$ \\
\hline & Median (IQR) & $111.5(27.5)$ & $1.2(0.4)$ & $1.2(0.3)$ & $32.1(10.0)$ & $24.0(5.8)$ \\
\hline & p-value (VS walking) & $0.013^{*}$ & $0.005^{\star}$ & $0.005^{\star}$ & $0.005^{\star}$ & $0.005^{\star}$ \\
\hline \multirow[t]{3}{*}{ Stride-on } & Mean (SD) & $93.8(26.5)$ & $0.6(0.2)$ & $0.6(0.2)$ & $18.8(6.3)$ & $16.8(5.0)$ \\
\hline & Median (IQR) & $89.0(28.3)$ & $0.6(0.2)$ & $0.5(0.2)$ & $17.1(5.5)$ & $18.0(6.3)$ \\
\hline & p-value (VS walking) & 0.959 & 0.332 & 0.575 & $0.005^{*}$ & 0.633 \\
\hline
\end{tabular}

Table 2: Summary of Results In Comparison to Walking Without Aid ("statistically significant values). SD, Standard Deviation. IQR, Interquartile Range 


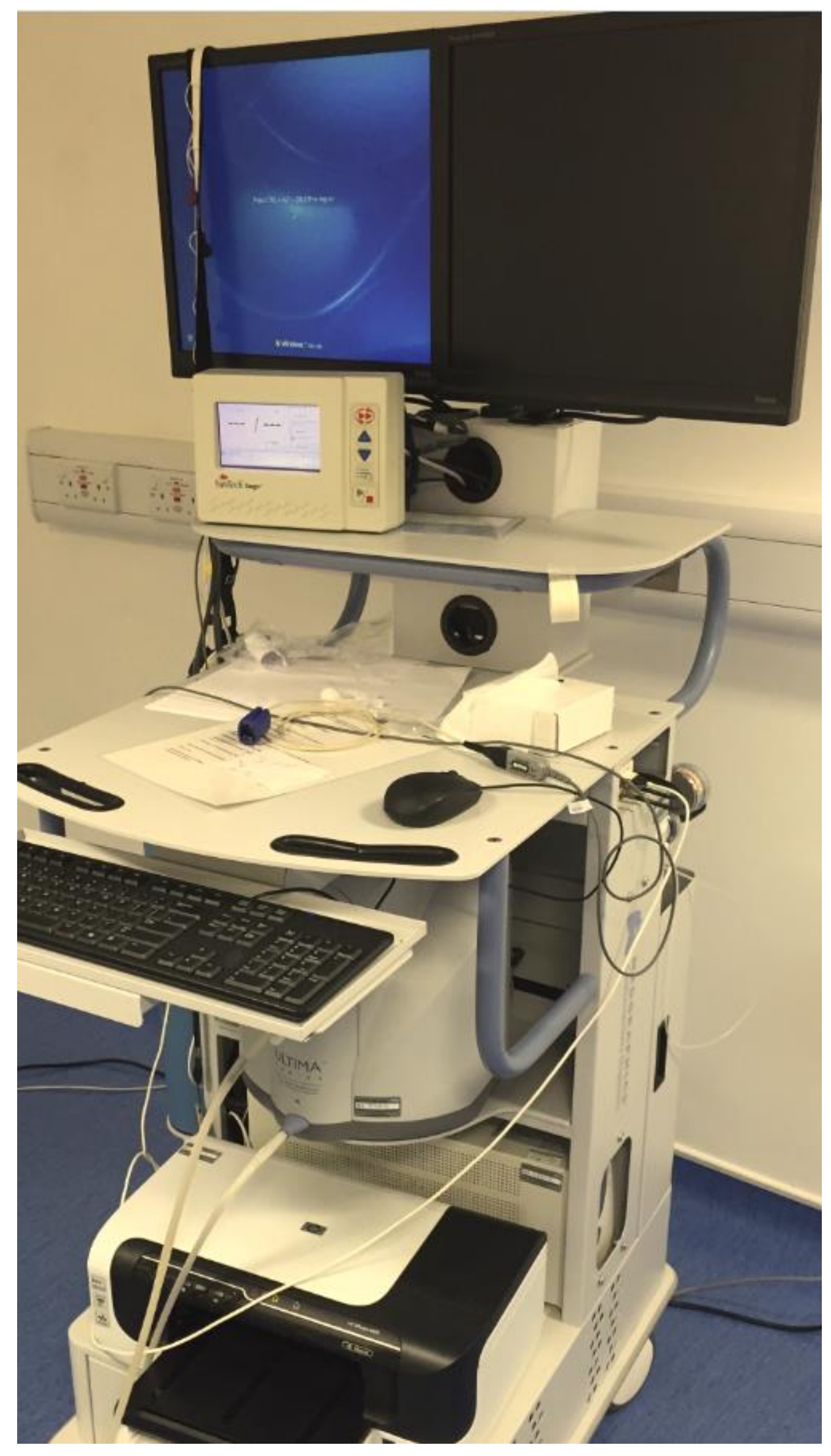




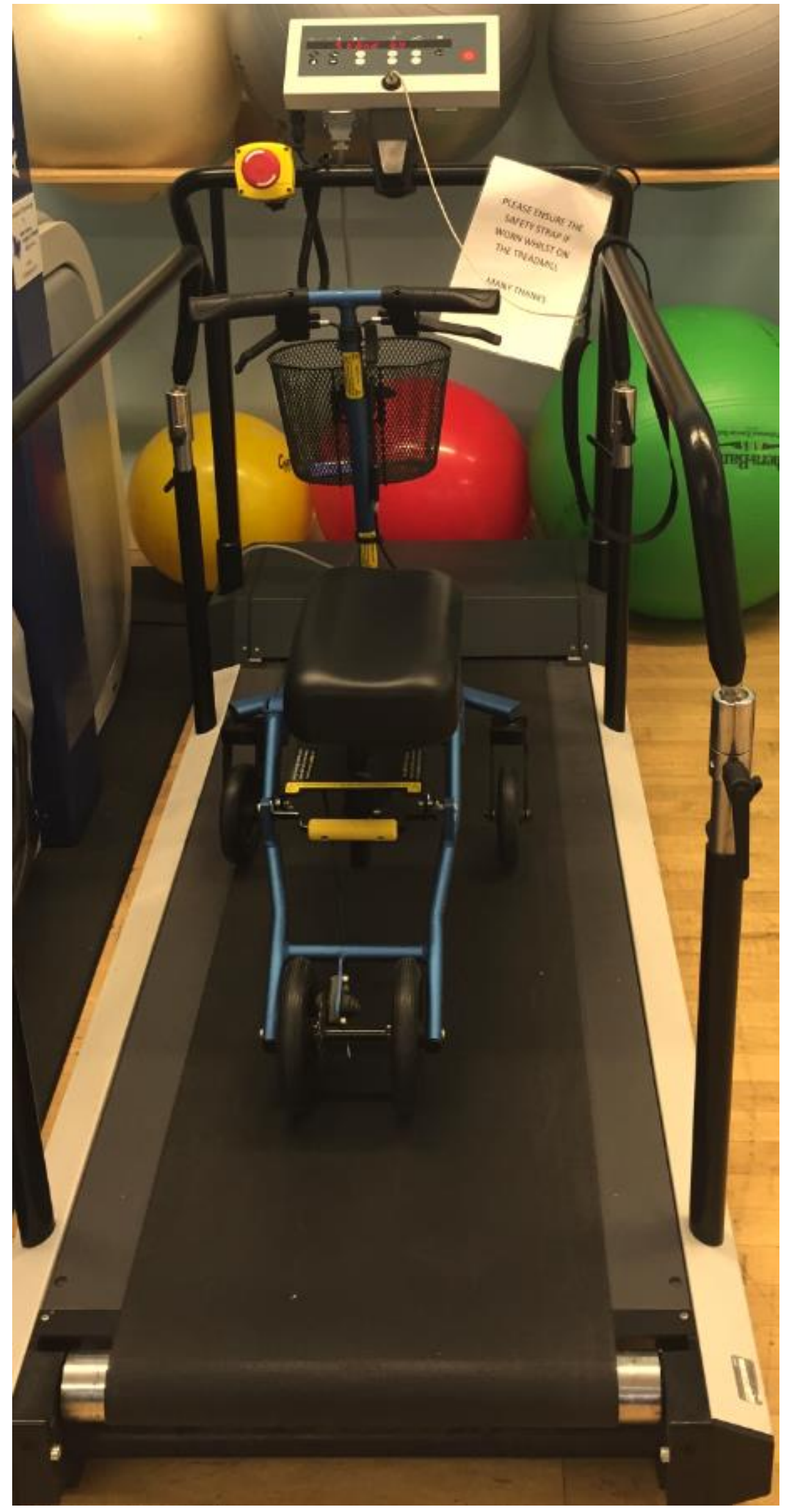

